\title{
Molecular Bars in Spiral Galaxies
}

\author{
Jean I. Turner \\ Department of Physics 8 Astronomy, UCLA, Los Angeles, CA \\ 90095-1562
}

\begin{abstract}
Images of molecular gas in spiral galaxies show two types of bar structure: large-scale bars, which are correlated with the presence of stellar bars, and generally follow the spiral structure, and nuclear "bars" of a few hundred parsecs in size, which show no direct correlation with the presence of a stellar bar. The dynamical origin of nuclear molecular bars is unclear, although they are consistent with radial inflow of gas into the nucleus, and subsequent evolution of the gas structure.
\end{abstract}

\section{Introduction}

Molecular bars are not the same as stellar bars, although there may be some indirect connection between the two. Stellar bars are relatively well-defined features. Molecular bars are not, and in fact the properties of "molecular bars" are strongly dependent on the sizescale one is considering. Molecular bars found in galactic nuclei are compact and well-defined structures with sizescales of a few hundred parsecs, and are particularly intense in the CO line. The dynamical origins of these nuclear bars are as yet unclear. Larger scale disk molecular bars, on the other hand, correspond to the kpc sizescales more typical of stellar bars, tend to be oriented along the stellar bars, and are therefore more likely to be directly related to stellar bars.

Observations of molecular gas in galaxies are for the most part done using the $\mathrm{CO}$ line, and the assumptions implicit in these studies are worth mentioning here. $\mathrm{CO}$ is used to trace molecular gas because excitation requirements for $\mathrm{H}_{2}$ preclude its use as a tracer of cool $\left(\mathrm{T}_{\mathrm{K}}<50 \mathrm{~K}\right)$ gas. The conversion from optically thick $\mathrm{CO}$ intensity to $\mathrm{H}_{2}$ column density is done using the empirical CO "conversion factor" (Solomon et al. 1987). This conversion factor can be understood as resulting from molecular clouds with virialized internal motions in pressure equilibrium with their overlying atomic envelopes (Elmegreen 1989). The $\mathrm{CO}$ conversion factor appears to be a reliable estimator of molecular mass in the Galaxy and on global scales in other large spiral galaxies (Solomon et al. 1987; Devereux \& Young 1990). The behavior of the conversion factor is less well known in regions of non-solar metallicity. $\mathrm{CO}$ is known to be a poor tracer of molecular mass in low metallicity galaxies, such as the Magellanic Clouds (Israel et al. 1986; Cohen et al. 1988; Maloney \& Black 1988). In high-metallicity regions, however, where the $\mathrm{CO}$ is optically thick, it is expected to be good tracer of molecular mass, and in fact, a relatively metallicity-independent one (Maloney 1990). This contention is supported by extragalactic ${ }^{13} \mathrm{CO}$ observations of 
nearby galaxies (Hurt \& Turner 1991; Turner \& Hurt 1992) and observations of the Galactic center cloud Sgr B2 (Lis \& Goldsmith 1989). In the discussion that follows I will adopt the Galactic $\mathrm{I}_{\mathrm{CO}} / \mathrm{H}_{2}$ conversion factor. This conversion factor should be good for all but the outermost reaches of large spiral galaxies.

Our knowledge of the molecular structure of galaxies is also limited by the resolution and imaging capabilities of millimeter telescopes. Single dish observations typically have resolutions of $20^{\prime \prime}$ to $60^{\prime \prime}$ at the frequency of the $\mathrm{J}=1-0$ transition of $\mathrm{CO}$, and typically have no imaging capability. Thus largescale images of molecular gas in galaxies are rare. Interferometers provide high resolution $\left(1^{\prime \prime}-3^{\prime \prime}\right)$ images but are also constrained by primary beamsizes of $20^{\prime \prime}$ $60^{\prime \prime}$ for the full image size. In addition, interferometers are sensitive to only the brightest $\mathrm{CO}$ emission, such as the emission from spiral arms, but generally fail to detect low-intensity, extended interarm emission. Another complication is that interferometer maps are insensitive to structures larger than their largest single fringe, which for millimeter telescopes corresponds to sizescales of $\sim 20^{\prime \prime}$. For emission more extended than this, one must add in single dish data, which is restricted by the above constraints. As a result, we know a fair amount about the structure of gas on sizescales of tens of parsecs in galactic nuclei, but little about the extended structure in spiral disks.

\section{Global Properties of Molecular Gas in Spirals}

Molecular gas in normal spiral galaxies is an inner galaxy phenomenon. Radial distributions of $\mathrm{H}_{2}$ surface densities are generally exponential (Young \& Scoville 1991). Central surface densities can exceed $10^{22} \mathrm{H}_{2} \mathrm{~cm}^{-2}$, or $160 \mathrm{M}_{\odot} \mathrm{pc}^{-2}$, averaged over half $\mathrm{kpc}$ sizescales. There is tremendous scatter in these values, however: for comparison, the central kpc of M31 has a surface density less than $4 \times 10^{19} \mathrm{H}_{2} \mathrm{~cm}^{-2}$. Morphological type is not a predictor of the molecular gas content of galaxies, although there is some indication that earlier types of spirals have higher $\mathrm{M}_{\mathrm{H}_{2}} / \mathrm{M}_{\mathrm{HI}}$ ratios (Young \& Knezek 1989).

Although there is little evidence that large-scale galactic structure is related to molecular gas content, there is an indication that star formation efficiencies are somewhat higher in barred spirals than in unbarred spirals. In their analysis of the Five College Radio Astronomy Observatory extragalactic CO survey, Young et al. (1995a, b) find that the ratio of infrared luminosity to molecular mass, often used as an indicator of star formation efficiency, is roughly a factor of two higher in barred galaxies than it is in unbarred galaxies. Given that the full range of $\mathrm{L}_{\mathrm{IR}} / \mathrm{M}_{\mathrm{H}_{2}}$ is more than two orders of magnitude, there are clearly other factors affecting star formation efficiency in galaxies. Still, barred galaxies, for whatever reason, seem to be a factor of two more efficient at making stars out of molecular gas than their unbarred counterparts.

\section{Nuclear Molecular Bars}

When the term "molecular bar" is used, it usually means a barlike feature of $<1 \mathrm{kpc}$ extent located in the central region of a spiral galaxy. The term is somewhat of a misnomer, because although these nuclear structures are barlike in appearance, there is no evidence that these "bars" are related in any sense 


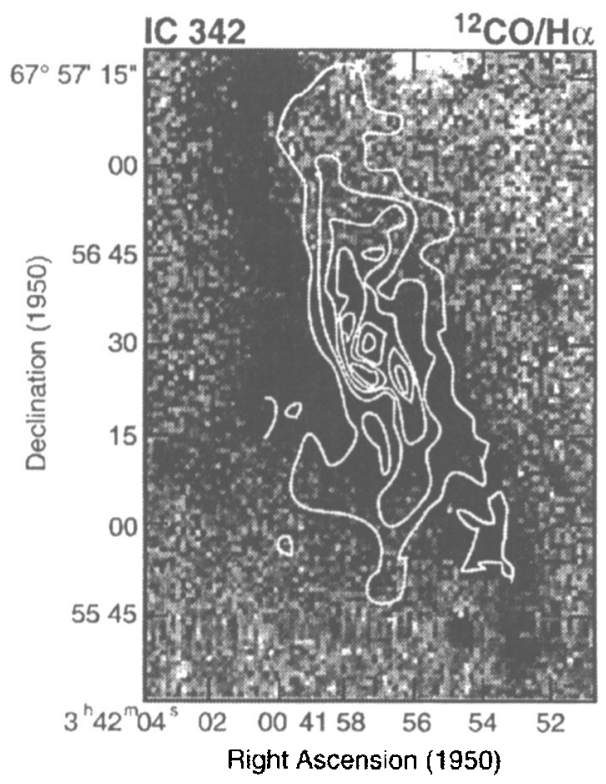

Figure 1. $\mathrm{CO}$ (contours; Levine et al. 1995) and $\mathrm{H} \alpha$ (greyscale; J. Young, private comm.) emission in the nucleus of IC 342.

to the standard galaxy bar classification. These nuclear "bars" have been the target of millimeter interferometers for over a decade (see Young \& Scoville 1991 for a partial list). To illustrate the properties of nuclear "bars", I shall focus on the closest of these bars beyond the Galaxy, in the nearby spiral IC 342 .

IC 342 is a large Scd galaxy just beyond the Local Group, at a distance of $1.8 \mathrm{Mpc}$. It has a large nuclear star-forming complex of luminosity $\sim 6 \times 10^{8} \mathrm{~L}_{\odot}$ (Becklin et al. 1980), located within $50 \mathrm{pc}$ of the dynamical center. The nuclear $\mathrm{CO}$ emission extends out to a radius of $\sim 500 \mathrm{pc}$ from the center, with a bar-like morphology (Lo et al. 1984). A recent map of the CO emission in IC 342, which consists of a mosaic of five pointings made with the Owens Valley Millimeter Interferometer, is shown in Figure 1 (Levine, Turner, \& Hurt 1995).

At $3^{\prime \prime}$ resolution the CO "bar" resolves into two open spiral arms of gas (Ishizuki et al. 1990) that continue to within $50 \mathrm{pc}$ of the dynamical center, with the nuclear starburst located at the inner end of the southern arm.

The nuclear arms in IC 342 are seen in $\mathrm{H} \alpha$ as well as in $\mathrm{CO}$ (J. Young, private communication; Figure 1, greyscale). The structure of the $\mathrm{H} \alpha$ arms is similar to that of the $\mathrm{CO}$ arms, but the $\mathrm{H} \alpha$ arms are offset from the $\mathrm{CO}$ arms by about $50-100 \mathrm{pc}$, with the $\mathrm{H} \alpha$ arms lying systematically to the outer, convex portion of the $\mathrm{CO}$ arms. Turner \& Hurt (1992) suggested, on the basis of the observed pattern and the kinematics of this gas, that the $\mathrm{CO}$ arms represent the gas response to a trailing spiral density wave. In this picture, the $\mathrm{CO}$ is 
brightest in the compression portion of the density wave, while $\mathrm{H} \alpha$ traces the stars that have formed after the density wave has passed. A similar pattern, with $\mathrm{CO}$ arms trailing the bright stellar arms, is seen in the nuclear region of NGC 6946 (Regan \& Vogel 1995).

The kinematics of the $\mathrm{CO}$ in IC 342 also support a density wave hypothesis. Strong radial motions are observed in IC 342 (Lo et al. 1984, Ishizuki et al. 1990). These motions are consistent with streaming motions of $40-50 \mathrm{~km} \mathrm{~s}^{-1}$ along the arm. Both the morphology and kinematics of the gas are very similar to what is observed in the density wave in the outer spiral arms of M51 (Vogel, Kulkarni, \& Scoville 1988).

Because of the high molecular mass fraction in IC 342, which approaches $50 \%$ in the inner $100 \mathrm{pc}$, this density wave may be gaseous and not stellar (Turner \& Hurt 1992). High molecular mass fractions of $40 \%$ or greater appear to be common in the inner few hundred parsecs of gas-rich galaxies (e.g., Canzian, Mundy, \& Scoville 1988; Hurt \& Turner 1991; Turner \& Hurt 1992; Kenney et al. 1993; Scoville et al. 1993). This result is indicated by both CO and ${ }^{13} \mathrm{CO}$ observations, which represent independent methods for determining masses. High molecular masses are also required by the large amounts of star formation observed in the nuclei of these galaxies. If the high molecular mass fractions are real, gas may drive the dynamics in the nuclear regions of gas-rich galaxies, rather than stars.

In any case, the molecular structures in gas-rich galactic nuclei are nearly always very non-axisymmetric in structure and are therefore likely to be evolutionary. Numerical simulations suggest that the dissipative and strongly shearing flows observed in nuclear molecular "bars", such as the one in IC 342, are likely to drive a slow radial inflow of gas into the nucleus (Roberts, Huntley, \& van Albada 1979; Athanassoula 1992). If the inflow rate is a few percent of the orbital motion as indicated by these simulations, the inflow rate inferred for IC 342 would be $\sim 1 \mathrm{~km} \mathrm{~s}^{-1}$. This inflow rate is not only sufficiently rapid to fuel the nuclear star formation at the present high rate, but would also deplete the CO within the inner kpc region within $\sim 5 \times 10^{8}$ years. This relatively short timescale suggests that the present gas-rich nucleus in IC 342 is only a transient phase.

\section{Large-Scale Molecular Bars}

Large-scale bars are not as well studied as nuclear "bars" because the mapping of large regions with single dish telescopes is very time-consuming. Fewer than half a dozen galaxies have large fully-sampled $\mathrm{CO}$ maps. The arcminute resolutions of single dish maps also make comparison with optical or near-infrared images of the stellar distribution difficult. However, it is these bars, not the nuclear "bars", that are likely to be directly driven by the stellar bars, and which may provide the link between the outer disk gas and the nuclear gas, in the event of interaction-driven gas inflow.

Large-scale maps of the unbarred spiral galaxy M101 have recently been done in both single dish (Kenney, Scoville, \& Wilson 1991), and interferometric (Kenney et al. 1995) modes. The single dish map shows that CO fills the disk of the galaxy, between the arms as well as in the arms, out to a radial distance 

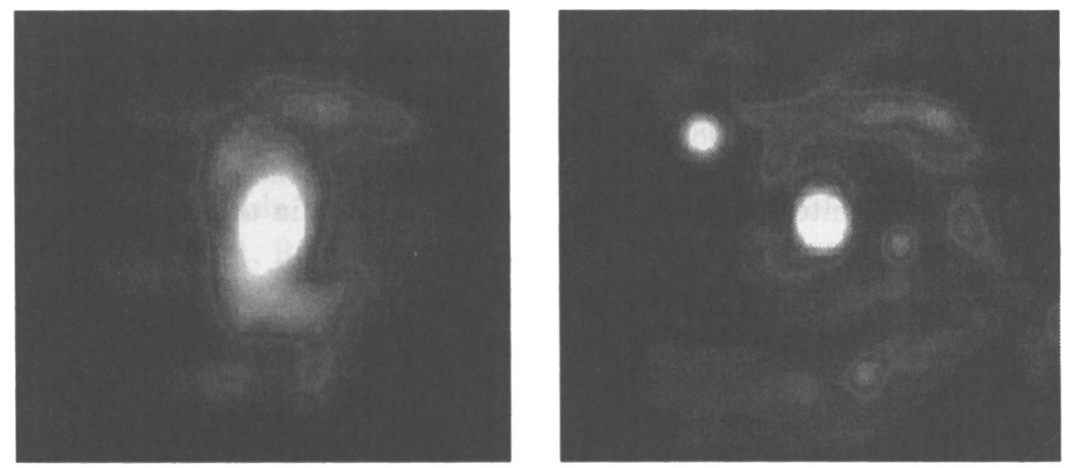

Figure 2. (Left) Single dish map of CO emission in IC 342 (Hurt et al. 1995). The size of the map is $15^{\prime}$ by $15^{\prime}$. (Right) $20 \mathrm{~cm}$ map of IC 342 (Condon 1987). The $20 \mathrm{~cm}$ emission is a rough indicator of star formation, and is fairly well correlated with the optical emission. Comparison of the $\mathrm{CO}$ and $20 \mathrm{~cm}$ images indicates that there are regions of high $\mathrm{CO}$ intensity that do not have correspondingly high star formation rates. These regions tend to lie within the bar region, which is evident in the $\mathrm{CO}$ map. The intense off-nuclear point source in the $20 \mathrm{~cm}$ map is a background radio galaxy.

of nearly $5^{\prime}$, or $8 \mathrm{kpc}$. The interferometric map shows $\mathrm{CO}$ in the arms only, indicating that the gas in the spiral arms is brighter than the interarm gas (Kenney et al. 1995). Although there is not an obvious stellar bar in M101, there is a weak oval distortion apparent in the CO. There is also a distinct nuclear molecular "bar", which has no obvious correlation with the larger-scale oval distortion.

The barred galaxy M83 has been mapped in the nuclear region by Handa et al. (1990) and a recent larger map has been done by Turner et al. (1995). As is the case for M101, the outer CO tends to generally follow the stellar structure, including the large-scale bar. There is also a nuclear molecular "bar" in M83. Although the large-scale stellar and CO bars are clearly correlated, the nuclear bar appears to have no obvious morphological link to the large-scale bars. The nuclear bar in M83, at position angle (deprojected) $\sim 0-10^{\circ}$, shows no special orientation with respect to the large-scale bar, at position angle $30^{\circ}$.

Fully-sampled single dish maps have also been made of IC 342 , although the proximity of this galaxy makes it difficult to map a large spatial area. The inner $3 \mathrm{kpc}$ nuclear region was been mapped by Sage \& Solomon (1991). A large scale map $\left(15^{\prime} \times 15^{\prime}\right.$, or $\left.8 \mathrm{kpc} \times 8 \mathrm{kpc}\right)$ of the $\mathrm{CO}$ emission has recently been completed for IC 342 (Hurt et al. 1995; Figure 2).

There is a hint of a weak stellar bar or perhaps a "lens" feature in IC 342, at position angle $-30^{\circ}$ (Levine et al. 1994). As in M83, the large-scale CO distribution does follow this weak bar, although the $\mathrm{CO}$ bar is more pronounced than the stellar bar. This large-scale bar, which covers $10^{\prime}$, or $6 \mathrm{kpc}$ extent, 
is displaced by about $30^{\circ}$ from the nuclear "bar", which covers the inner half $\mathrm{kpc}$. Comparison with the radio continuum image of the galaxy indicates that although there is plentiful $\mathrm{CO}$ in the bar, the star formation appears to be suppressed in the bar region, as compared with either the nucleus or the spiral arms (Hurt et al.). The inhibition of star formation within the region of a bar was attributed by Tubbs (1980) to high streaming motions within the gas of the bar region, but streaming motions are not apparent on these large sizescales in IC 342. It will be interesting to see if this result holds in other, more strongly barred galaxies, as well.

Acknowledgments. I would like to thank Judy Young, Deborah Levine, and Robert Hurt for providing results in advance of publication. This research is supported by NSF Grant 9417968 .

\section{References}

Athanassoula, E. 1992, MNRAS, 259, 345

Becklin, E. E., Gatley, I., Matthews, K., Neugebauer, G., Sellgren, K., Werner, M. W., \& Wynn-Williams, C. G. 1980, ApJ, 236, 441

Cohen, R. S., Dame, T. M., Garay, G., Mantani, J., Rubio, M., \& Thaddeus, P. 1988, ApJ, 331, L95

Canzian, B., Mundy, L. G. \& Scoville, N. Z. 1988, ApJ, 333, 157

Condon, J. J. 1987, ApJS, 65, 485

Devereux, N. A. \& Young, J. S. 1990, ApJ, 371, 515

Elmegreen, B. G. 1989, ApJ, 338, 178

Handa, T., Nakai, N., Sofue, Y., Hayashi, M., \& Fujimoto, M. 1990, PASJ, 42, 1

Hurt, R. L., Levine, D. A., Martin, R. N., Turner, J. L., \& Ho, P. T. P. 1995, submitted

Hurt, R. L. \& Turner, J. L. 1991, ApJ, 377, 434

Ishizuki, S., Kawabe, R., Ishiguro, M., Okumura, S. K., Morita, K.-I., Chikada, Y., \& Kasuga, T. 1990, Nature, 344, 224

Israel, F. P., de Graauw, Th., van de Stadt, H. \& de Vries, C. P. 1986, ApJ, 303, 186

Kenney, J. D. P., Scoville, N. Z., \& Wilson, C. D. 1991, ApJ, 366, 432

Kenney, J. D. P., Scoville, N. Z., \& Wilson, C. D. 1995, in preparation

Kenney, J. D. P., Carlstrom, J. E., \& Young, J. S. 1993, ApJ, 418, 687

Levine, D. A., Hurt, R. L., \& Turner, J. L. 1994, in Infrared Astronomy with Arrays, I. McLean, Dordrecht: Kluwer, 181

Levine, D. A., Turner, J. L., \& Hurt, R. L. 1995, in preparation

Lis, D. C. \& Goldsmith, P. F. 1989, ApJ, 337, 704

Lo, K. Y. et al. 1984, ApJ, 282, L59

Maloney, P. 1990, ApJ, 348, L9

Maloney, P. \& Black, J. H. 1988, ApJ, 325, 389

Regan, M. \& Vogel, S. V. 1995, ApJ, in press 
Roberts, W., Huntley, J., \& van Albada, G. D. 1979, ApJ, 233, 67

Sage, L. J. \& Solomon, P. M. 1991, ApJ, 380, 392

Scoville, N. Z., Thakkar, D., Carlstrom, J. E., \& Sargent, A. I. 1993, ApJ, 404, I.59

Solomon, P. M., Rivolo, A. R., Barrett, J. W., \& Yahil, A. 1987, ApJ, 319, 730

Tubbs, A. D. 1982, ApJ, 255, 458

Turner, J. L., Buchholz, L., \& Martin, R. N. 1995, in preparation

Turner, J. L. \& Hurt, R. L. 1992, ApJ, 384, 72

Vogel, S. N., Kulkarni, S. V., \& Scoville, N. Z., Nature, 334, 402

Young, J. S. \& Knezek, P. M. 1989, ApJ, 347, L55

Young, J. S. et al. 1995a, ApJ, 98, 219

Young, J. S. et al. 1995b, in preparation

Young, J. S. \& Scoville, N. Z. 1991, ARA\&A, 29, 581

\section{Discussion}

T. Hawarden: In the FCRAO survey, the SB's have twice the $\mathrm{L}_{\mathrm{TR}} / \mathrm{M}_{\mathrm{H}_{2}}$ of the SA's. How was this sample chosen?

J. Turner: There were over 200 galaxies in this survey, and it was not a fluxlimited sample, I believe. I refer you to the Young et al. (1995) data paper in the May 1995 ApJ.

A. Zasov: Is the gas mass fraction high enough to account for the observed velocity of rotation without involving the dense bulge?

J. Turner: Yes.

A. Zasov: Can you tell something about the velocity dispersion of molecular clouds in circumnuclear regions? It may play a role in determining the star formation efficiency.

J. Turner: Velocity dispersions are difficult to determine: the gas motions are dominated by systematic motions, such as arm streaming and rotation, of order $50 \mathrm{~km} \mathrm{~s}^{-1}$. The presence of large amounts of shear in the gas within the nuclear arms probably explains why most of the star formation takes place not in the arms, but at the galactic center, where the arms end. 